\title{
HIGH EFFICIENCY GEARS SYNTHESIS BY AVOID THE INTERFERENCES
}

\author{
Florian Ion Tiberiu Petrescu \\ Bucharest Polytechnic University, Romania \\ E-mail: petrescuflorian@yahoo.com \\ Relly Victoria Virgil Petrescu \\ Bucharest Polytechnic University, Romania \\ E-mail: petrescuvictoria@yahoo.com
}

\section{ABSTRACT}

Submission: $27 / 09 / 2013$

Revision: 19/10/2013

Accept: 20/10/2013

The paper presents an original method to determine the efficiency of the gear, the forces of the gearing, the velocities and the powers. It is analyzing the influence of a few parameters concerning gear efficiency. These parameters are: $z_{1}$ - the number of teeth for the primary wheel of gear; $z_{2}$ - the number of teeth of the secondary wheel of gear; alpha $_{0}$ - the normal pressure angle on the divided circle; beta - the inclination angle. With the relations presented in this paper, it can synthesize the gear's mechanisms. Today, the gears are present everywhere, in the mechanical's world (In vehicle's industries, in electronics and electro-technique equipments, in energetically industries, etc). Optimizing this mechanism (the gears mechanism), we can improve the functionality of the transmissions with gears. At the gear mechanisms an important problem is the interference of the teeth. To avoid the interference between teeth, we must know the minimum number of teeth of the driving wheel, in function of the pressure angle (normal on the pitch circle, alpha0), in function of the tooth inclination angle (beta), and in function of the transmission ratio (i).

Keywords: gears; gear efficiency; forces; velocities; powers; number of teeth; avoid the interference 
INDEPENDENT JOURNAL OF MANAGEMENT \& PRODUCTION (IJM\&P)

http://www.ijmp.jor.br

v. 5, n. 2, February - May 2014.

ISSN: 2236-269X

DOI: 10.14807/ijmp.v5i2.147

\section{INTRODUCTION}

Gears, broke today in all fields. They have the advantage of working with very high efficiency. Additionally gears can transmit large loads. Regardless of their size, gear must be synthesized carefully considering the specific conditions. This paper tries to present the main conditions that must be met for correct synthesis of a gear (PETRESCU, 2012; PETRESCU; PETRESCU, 2002; PETRESCU; PETRESCU, 2003; PETRESCU; PETRESCU; POPESCU, 2007).

Top of the use of sprocket mechanisms must be sought in ancient Egypt with at least a thousand years before Christ. Here were used for the first time, transmissions wheeled "spurred" to irrigate crops and worm gears to the cotton processing (LIN C., 2011; LEI X., 2011). With 230 years BC, in the city of Alexandria in Egypt, they have been used the wheel with more levers and gear rack. Such gears have been constructed and used beginning from the earliest times, to the top for lifting the heavy anchors of vessels and for claim catapults used on the battlefields. Then, they were introduced in cars with wind and water (as a reducing or multiplying at the pump from windmills or water), (see Figure 1).

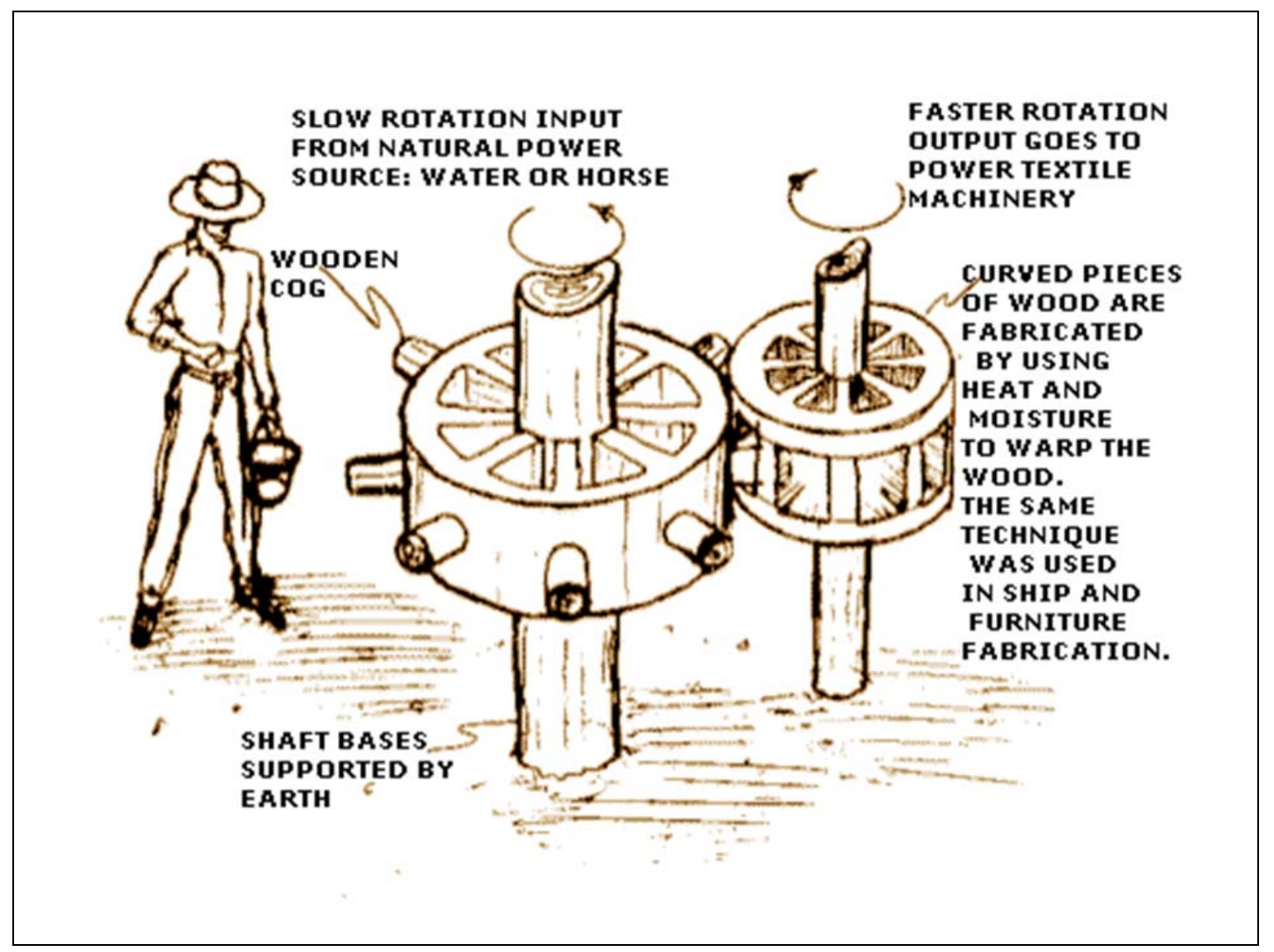

Figure 1: Transmissions wheeled "spurred" to irrigate crops and worm gears to the cotton processing 
The Antikythera Mechanism is the name given to an astronomical calculating device, measuring about 32 by 16 by $10 \mathrm{~cm}$, which was discovered in 1900 in a sunken ship just off the coast of Antikythera, an island between Crete and the Greek mainland. Several kinds of evidence point incontrovertibly to around 80 B.C. for the date of the shipwreck. The device, made of bronze gears fitted in a wooden case, was crushed in the wreck, and parts of the faces were lost, "the rest then being coated with a hard calcareous deposit at the same time as the metal corroded away to a thin core coated with hard metallic salts preserving much of the former shape of the bronze" during the almost 2000 years it lay submerged. (See Antikythera 1, in Figure 2).

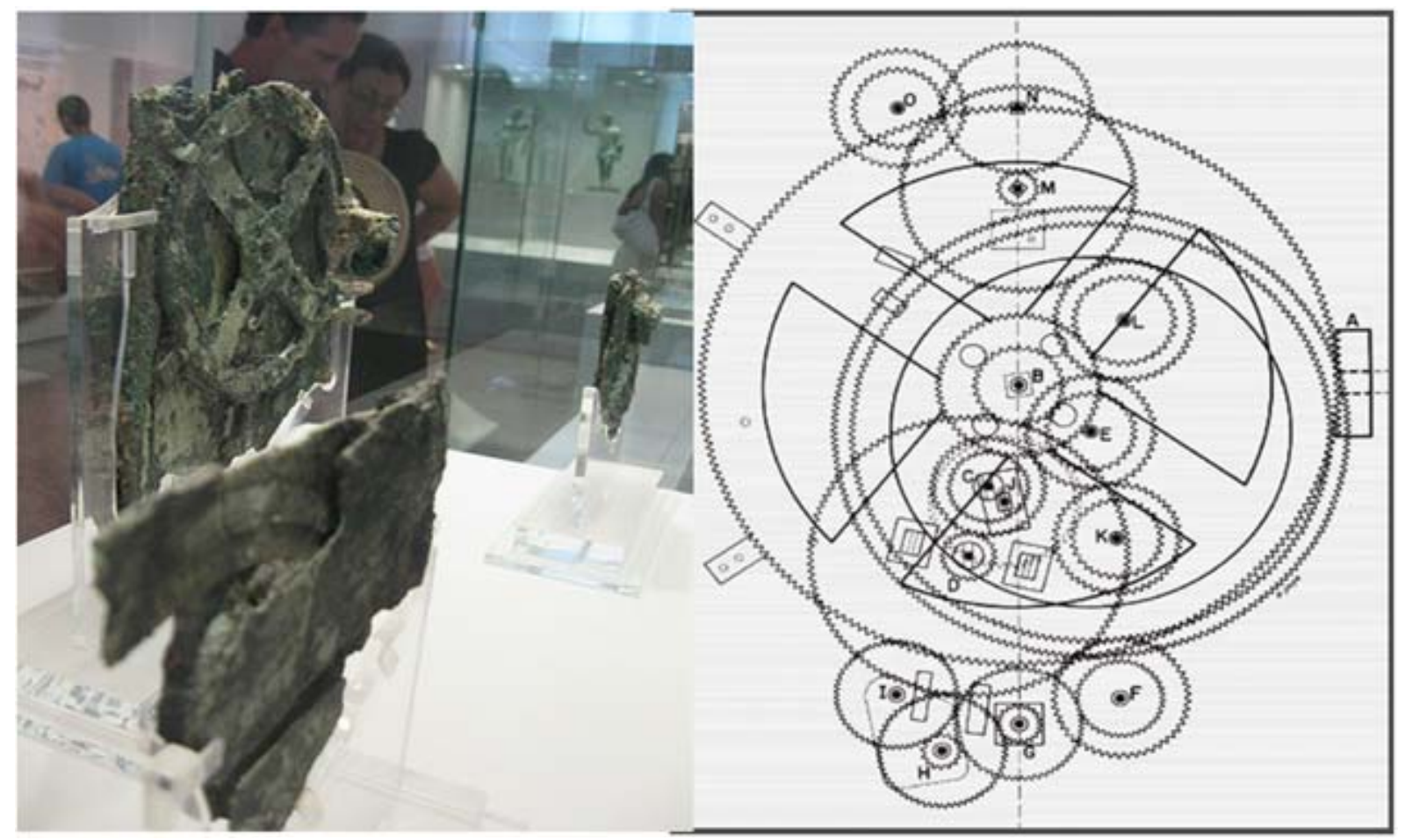

Figure 2: The Antikythera Mechanism is the name given to an astronomical calculating device

Modern adventure began with the gear wheel spurred of Leonardo da Vinci, in the fifteenth century. He founded the new kinematics and dynamics stating inter alia the principle of superposition of independent movements (Figure 3). 


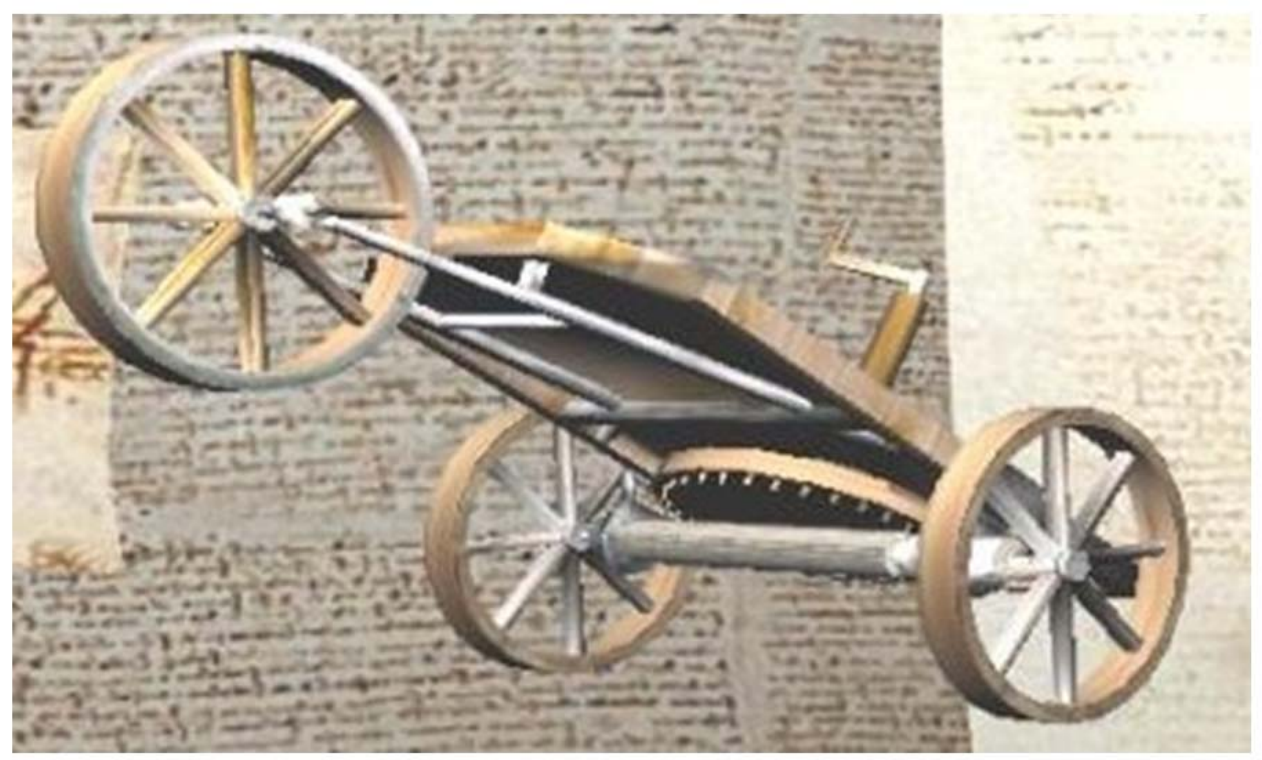

Figure 3: The modern adventure began with the gear wheel spurred of Leonardo da Vinci, in the fifteenth century

Benz had engine with transmissions sprocket gearing and Gear chain (patented after 1882, Figure 4), but the first gearing patent (the drawings of a patent first gear transmission) and of gearing wheels with chain was made in 1870 by the British Starley \& Hillman (REY, 2013).

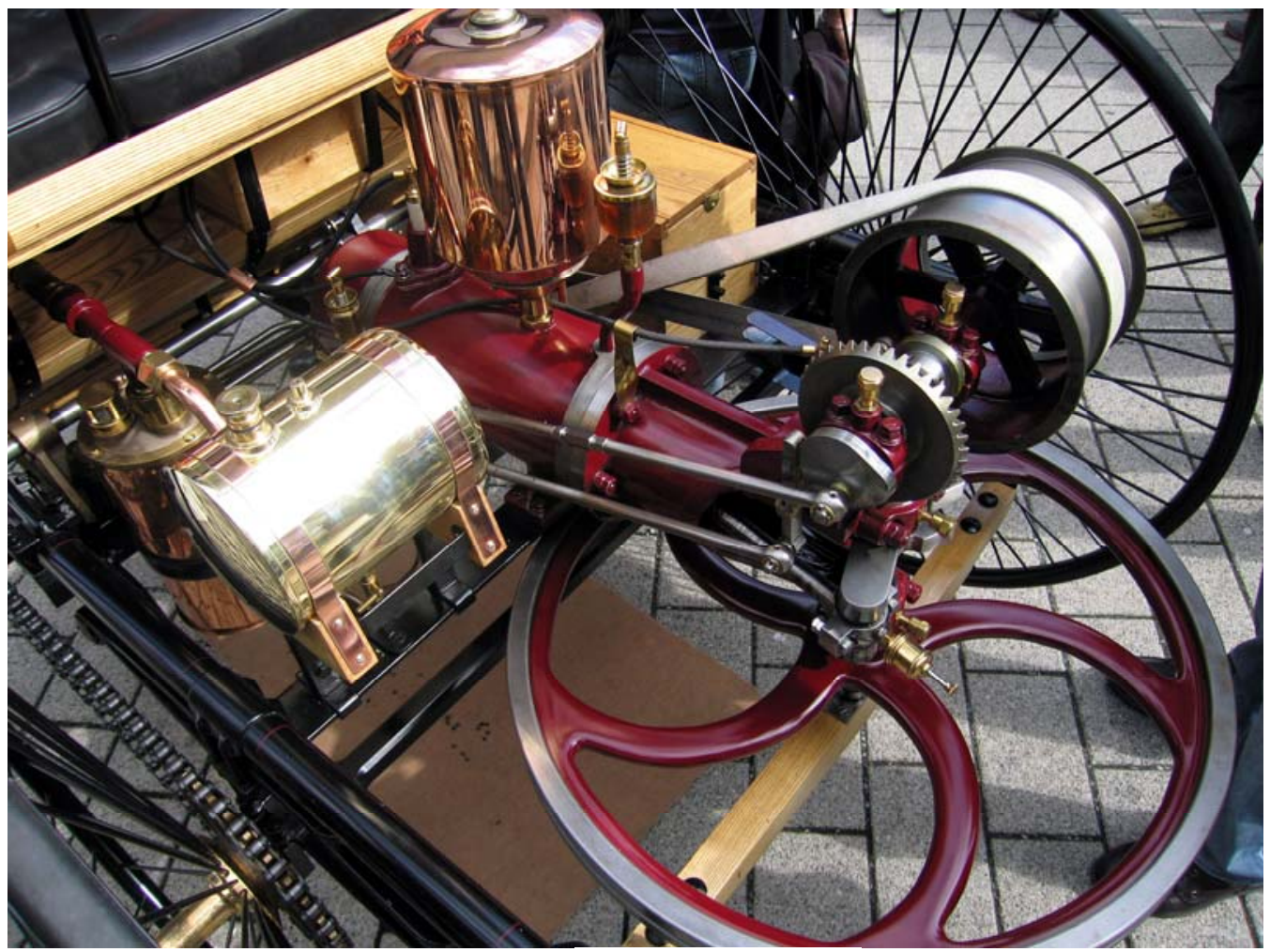

Figure 4: The Benz patent 
INDEPENDENT JOURNAL OF MANAGEMENT \& PRODUCTION (IJM\&P)

http://www.ijmp.jor.br

v. 5, n. 2, February - May 2014.

ISSN: 2236-269X

DOI: 10.14807/ijmp.v5i2.147

After 1912, in Cleveland (USA), begin to produce industrial specialized wheels and gears (cylindrical, worm, conical, with straight teeth, inclined or curved; see Figure 5).
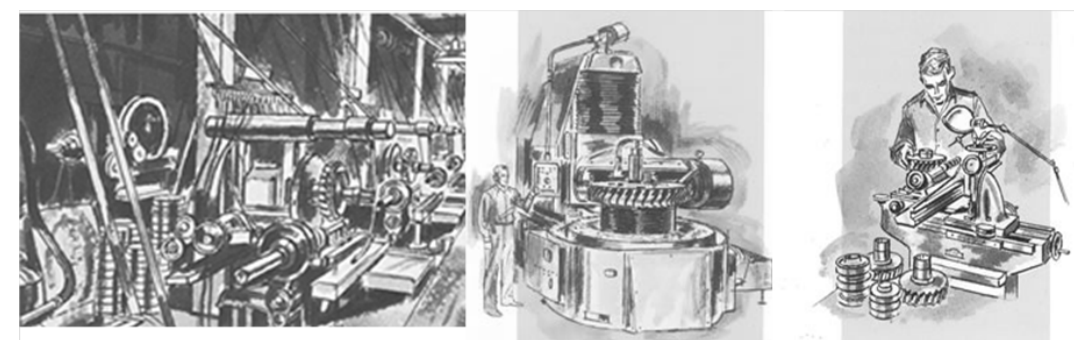

After 1912, in Cleveland (USA), begin to produce industrial specialized wheels and gears (cylindrical, worm,
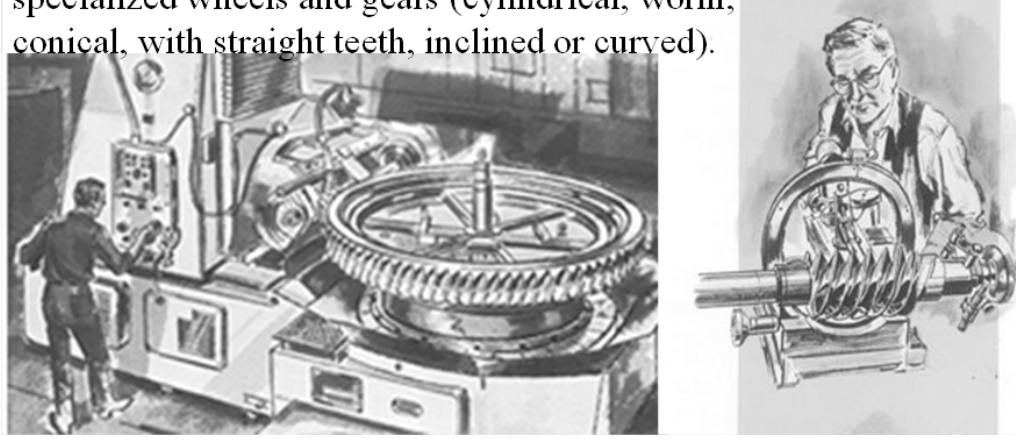

Figure 5: In Cleveland, after 1912 begin to produce industrial specialized wheels

Today, the gears are present everywhere, in the mechanical's world (In vehicle's industries, in electronics and electro-technique equipment's, in energetically industries, etc.; Figure 6).

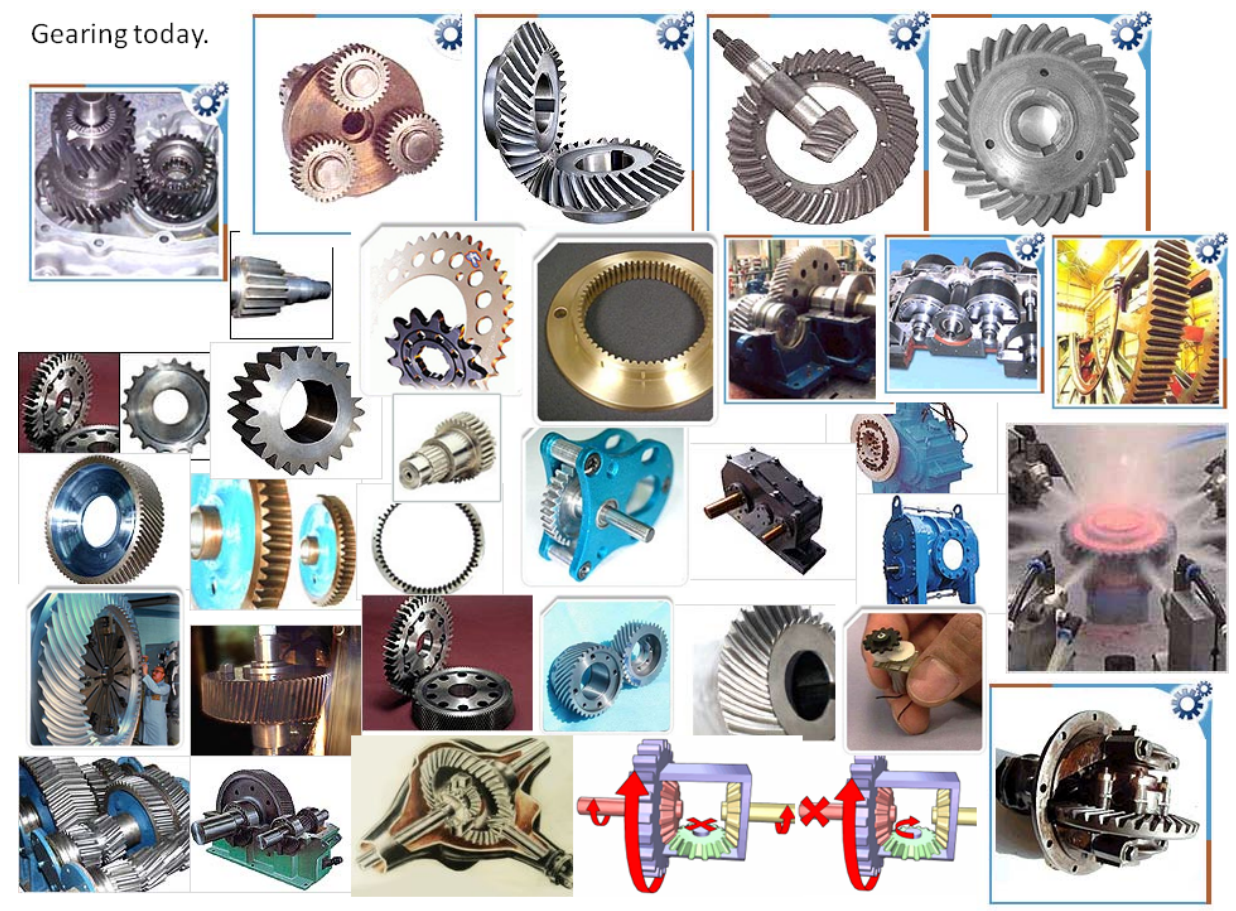

Figure 6. Gearing today 


\section{GEARS SYNTHESIS}

The calculating relations (PETRESCU, 2012), are the next (2.1-2.21).

\subsection{Determining the momentary dynamic (mechanical) efficiency, the forces of the gearing, and the velocities}

The forces, velocities, powers and efficiency can be obtained with the relationships 2.1-2.6; the forces from gearing may be seen in the Figure 7.

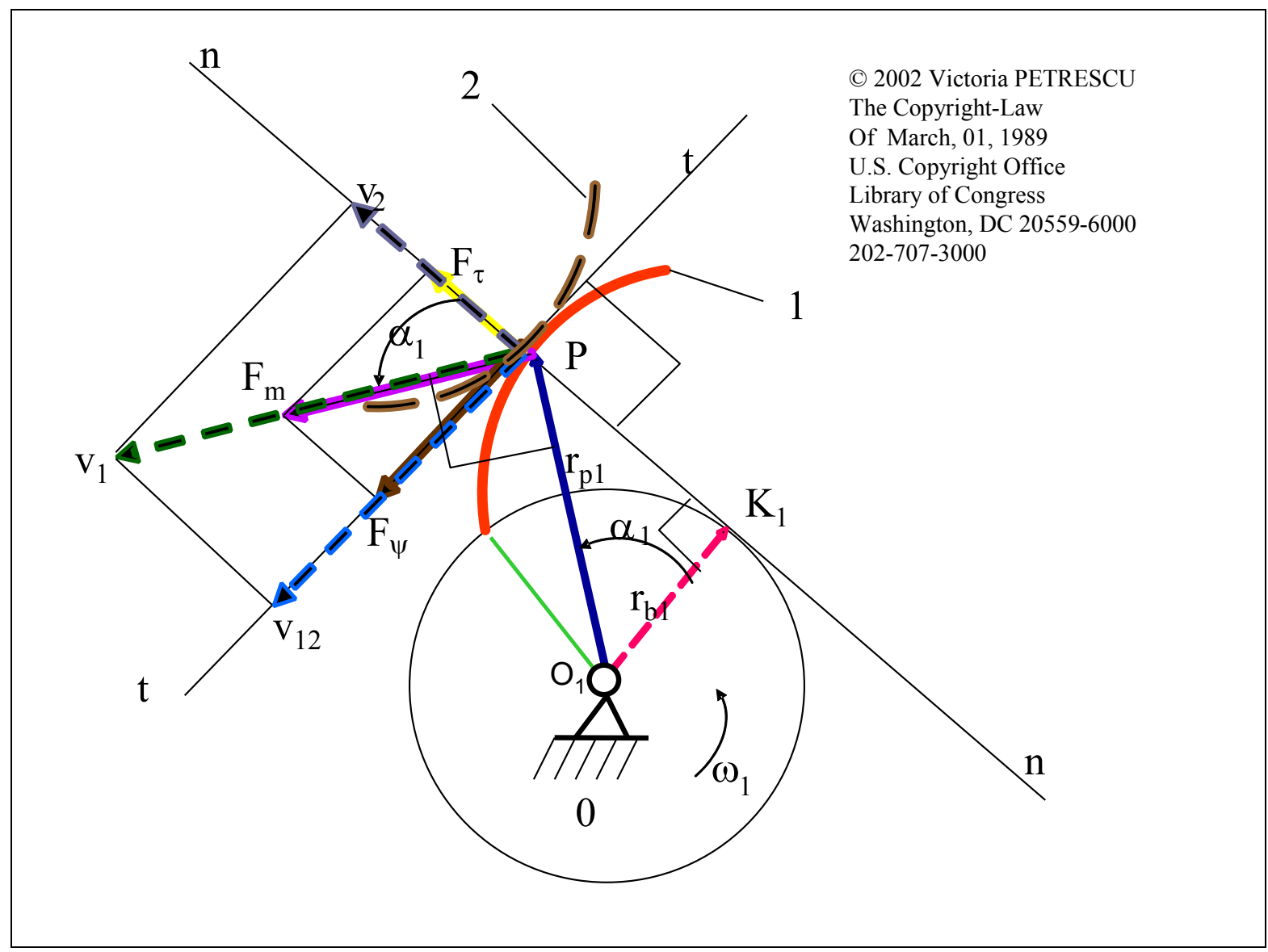

Figure 7: The forces and the velocities of the gearing

$$
\left\{\begin{array}{l}
F_{\tau}=F_{m} \cdot \cos \alpha_{1} \\
F_{\psi}=F_{m} \cdot \sin \alpha_{1} \\
v_{2}=v_{1} \cdot \cos \alpha_{1} \\
v_{12}=v_{1} \cdot \sin \alpha_{1} \\
\bar{F}_{m}=\bar{F}_{\tau}+\bar{F}_{\psi} \\
\bar{v}_{1}=\bar{v}_{2}+\bar{v}_{12}
\end{array}\right.
$$

with: $\quad F_{m}$ - the motive force (the driving force);

$F \tau$ - the transmitted force (the useful force);

$F \psi$ - the slide force (the lost force); 
$v_{1}$ - the velocity of element 1 , or the speed of wheel 1 (the driving wheel);

$v_{2}$ - the velocity of element 2 , or the speed of wheel 2 (the driven wheel);

$v_{12}$ - the relative speed of the wheel 1 in relation with the wheel 2 (this is a sliding speed).

The consumed power (in this case the driving power):

$$
P_{c} \equiv P_{m}=F_{m} \cdot v_{1}
$$

The useful power (the transmitted power from the profile 1 to the profile 2 ) will be written:

$$
P_{u} \equiv P_{\tau}=F_{\tau} \cdot v_{2}=F_{m} \cdot v_{1} \cdot \cos ^{2} \alpha_{1}
$$

The lost power will be written:

$$
P_{\psi}=F_{\psi} \cdot v_{12}=F_{m} \cdot v_{1} \cdot \sin ^{2} \alpha_{1}
$$

The momentary efficiency of couple will be calculated directly with the next relation:

$$
\left\{\begin{array}{l}
\eta_{i}=\frac{P_{u}}{P_{c}} \equiv \frac{P_{\tau}}{P_{m}}=\frac{F_{m} \cdot v_{1} \cdot \cos ^{2} \alpha_{1}}{F_{m} \cdot v_{1}} \\
\eta_{i}=\cos ^{2} \alpha_{1}
\end{array}\right.
$$

The momentary losing coefficient (PETRESCU, 2012), will be written:

$$
\left\{\begin{array}{l}
\psi_{i}=\frac{P_{\psi}}{P_{m}}=\frac{F_{m} \cdot v_{1} \cdot \sin ^{2} \alpha_{1}}{F_{m} \cdot v_{1}}=\sin ^{2} \alpha_{1} \\
\eta_{i}+\psi_{i}=\cos ^{2} \alpha_{1}+\sin ^{2} \alpha_{1}=1
\end{array}\right.
$$

It can easily see that the sum of the momentary efficiency and the momentary losing coefficient is 1 .

Now, one can determine the geometrical elements of gear. These elements will be used in determining the couple efficiency, $\eta$.

\subsection{The geometrical elements of the gear}

We can determine the next geometrical elements of the external gear, (for the right teeth, $\beta=0)$, (PETRESCU, 2012).

The radius of the basic circle of the wheel 1 (of the driving wheel), (2.7).

$$
r_{b 1}=\frac{1}{2} \cdot m \cdot z_{1} \cdot \cos \alpha_{0}
$$

The radius of the outside circle of wheel 1 (2.8): 


$$
r_{a 1}=\frac{1}{2} \cdot\left(m \cdot z_{1}+2 \cdot m\right)=\frac{m}{2} \cdot\left(z_{1}+2\right)
$$

It determines now the maximum pressure angle of the gear (2.9):

$$
\cos \alpha_{1 M}=\frac{r_{b 1}}{r_{a 1}}=\frac{\frac{1}{2} \cdot m \cdot z_{1} \cdot \cos \alpha_{0}}{\frac{1}{2} \cdot m \cdot\left(z_{1}+2\right)}=\frac{z_{1} \cdot \cos \alpha_{0}}{z_{1}+2}
$$

And now one determines the same parameters for the wheel 2 , the radius of basic circle (2.10) and the radius of the outside circle (2.11) for the wheel 2 :

$$
\begin{gathered}
r_{b 2}=\frac{1}{2} \cdot m \cdot z_{2} \cdot \cos \alpha_{0} \\
r_{a 2}=\frac{m}{2} \cdot\left(z_{2}+2\right)
\end{gathered}
$$

Now it can determine the minimum pressure angle of the external gear (2.12, 2.13):

$$
\left\{\begin{array}{l}
\operatorname{tg} \alpha_{1 m}=\frac{N}{r_{b 1}} \\
N=\left(r_{b 1}+r_{b 2}\right) \cdot \operatorname{tg} \alpha_{0}-\sqrt{r_{a 2}^{2}-r_{b 2}^{2}}= \\
=\frac{1}{2} \cdot m \cdot\left(z_{1}+z_{2}\right) \cdot \sin \alpha_{0}- \\
-\frac{m}{2} \cdot \sqrt{\left(z_{2}+2\right)^{2}-z_{2}^{2} \cdot \cos ^{2} \alpha_{0}}= \\
=\frac{m}{2} \cdot\left[\left(z_{1}+z_{2}\right) \cdot \sin \alpha_{0}-\right. \\
\left.-\sqrt{z_{2}^{2} \cdot \sin ^{2} \alpha_{0}+4 \cdot z_{2}+4}\right] \\
\operatorname{tg} \alpha_{1 m}=\left[\left(z_{1}+z_{2}\right) \cdot \sin \alpha_{0}-\sqrt{z_{2}^{2} \cdot \sin ^{2} \alpha_{0}+4 \cdot z_{2}+4}\right] /\left(z_{1} \cdot \cos \alpha_{0}\right)
\end{array}\right.
$$

Now we can determine, for the external gear, the minimum (2.13) and the maximum (2.9) pressure angle for the right teeth. For the external gear with bended teeth $(\beta \neq 0)$ it uses the relations $(2.14,2.15$ and 2.16$)$ :

$$
\begin{gathered}
\operatorname{tg} \alpha_{t}=\frac{\operatorname{tg} \alpha_{0}}{\cos \beta} \\
\operatorname{tg} \alpha_{1 m}=\left[\left(z_{1}+z_{2}\right) \cdot \frac{\sin \alpha_{t}}{\cos \beta}-\sqrt{z_{2}^{2} \cdot \frac{\sin ^{2} \alpha_{t}}{\cos ^{2} \beta}+4 \cdot \frac{z_{2}}{\cos \beta}+4}\right] \cdot \frac{\cos \beta}{z_{1} \cdot \cos \alpha_{t}}
\end{gathered}
$$




$$
\cos \alpha_{1 M}=\frac{\frac{z_{1} \cdot \cos \alpha_{t}}{\cos \beta}}{\frac{z_{1}}{\cos \beta}+2}
$$

For the internal gear with bended teeth $(\beta \neq 0)$ it uses the relations (2.14 with 2.17, 2.18-A, or with 2.19, 2.20-B):

A. When the driving wheel 1 , has external teeth:

$$
\begin{gathered}
\operatorname{tg} \alpha_{1 m}=\left[\left(z_{1}-z_{2}\right) \cdot \frac{\sin \alpha_{t}}{\cos \beta}+\sqrt{\left.z_{2}^{2} \cdot \frac{\sin ^{2} \alpha_{t}}{\cos ^{2} \beta}-4 \cdot \frac{z_{2}}{\cos \beta}+4\right]} \cdot \frac{\cos \beta}{z_{1} \cdot \cos \alpha_{t}}\right. \\
\cos \alpha_{1 M}=\frac{\frac{z_{1} \cdot \cos \alpha_{t}}{\frac{\cos \beta}{\cos \beta}+2}}{}
\end{gathered}
$$

B. When the driving wheel 1, have internal teeth:

$$
\begin{gathered}
\operatorname{tg} \alpha_{1 M}=\left[\left(z_{1}-z_{2}\right) \cdot \frac{\sin \alpha_{t}}{\cos \beta}+\sqrt{z_{2}^{2} \cdot \frac{\sin ^{2} \alpha_{t}}{\cos ^{2} \beta}+4 \cdot \frac{z_{2}}{\cos \beta}+4}\right] \cdot \frac{\cos \beta}{z_{1} \cdot \cos \alpha_{t}} \\
\cos \alpha_{1 m}=\frac{\frac{\frac{z_{1} \cdot \cos \alpha_{t}}{\cos \beta}}{\frac{z_{1}}{\cos \beta}-2}}{}
\end{gathered}
$$

\subsection{Determining the efficiency of the gear}

The efficiency of the gear will be calculated through the integration of momentary efficiency on all sections of gearing movement, namely from the minimum pressure angle to the maximum pressure angle, the relation (2.21), (PETRESCU, 2012).

$$
\begin{aligned}
& \eta=\frac{1}{\Delta \alpha} \cdot \int_{a_{m}}^{\alpha_{M}} \eta_{i} \cdot d \alpha=\frac{1}{\Delta \alpha} \int_{\alpha_{m}}^{\alpha_{M}} \cos ^{2} \alpha \cdot d \alpha=\frac{1}{2 \cdot \Delta \alpha} \cdot\left[\frac{1}{2} \cdot \sin (2 \cdot \alpha)+\alpha\right]_{\alpha_{m}}^{\alpha_{M}}= \\
& =\frac{1}{2 \cdot \Delta \alpha}\left[\frac{\sin \left(2 \alpha_{M}\right)-\sin \left(2 \alpha_{m}\right)}{2}+\Delta \alpha\right]=\frac{\sin \left(2 \cdot \alpha_{M}\right)-\sin \left(2 \cdot \alpha_{m}\right)}{4 \cdot\left(\alpha_{M}-\alpha_{m}\right)}+0.5
\end{aligned}
$$

\subsection{External and internal gearing}

Exterior gearing are the most common, not because it would be best but because they are easier to designed and constructed (Figure 8). Interior gearing may be more efficient and more reliable when they are projected properly (Figure 9). 


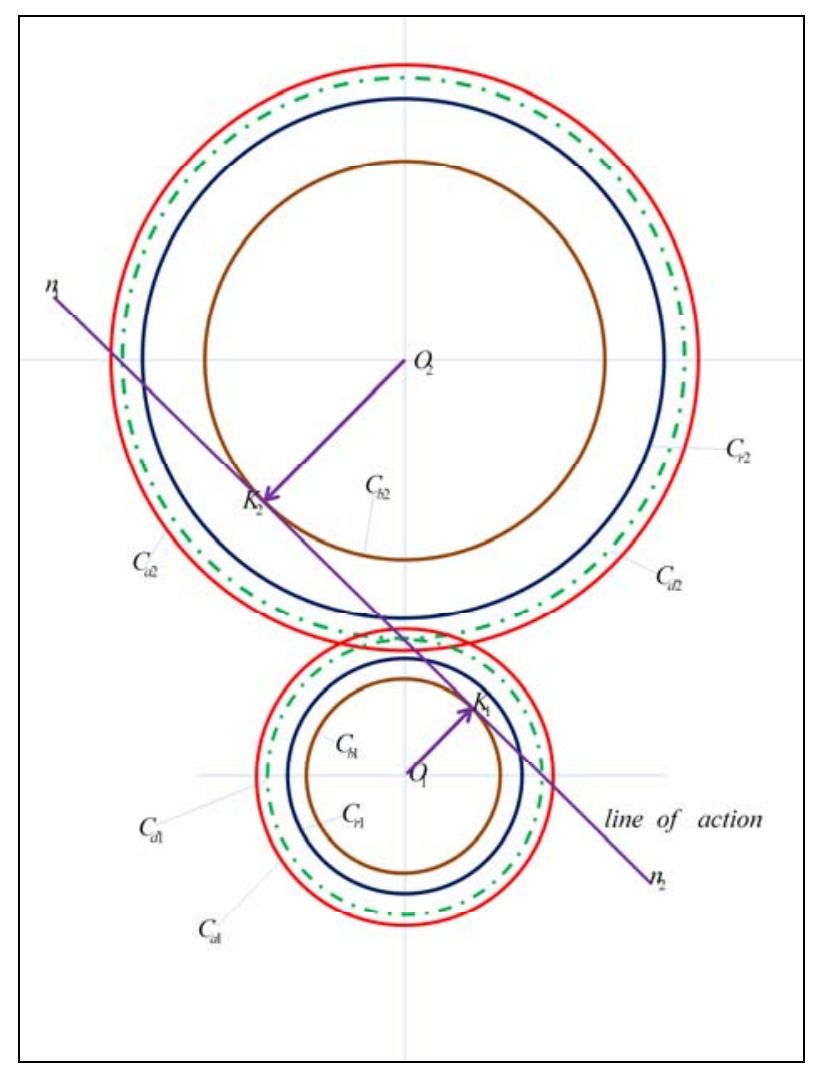

Figure 8. An external gearing

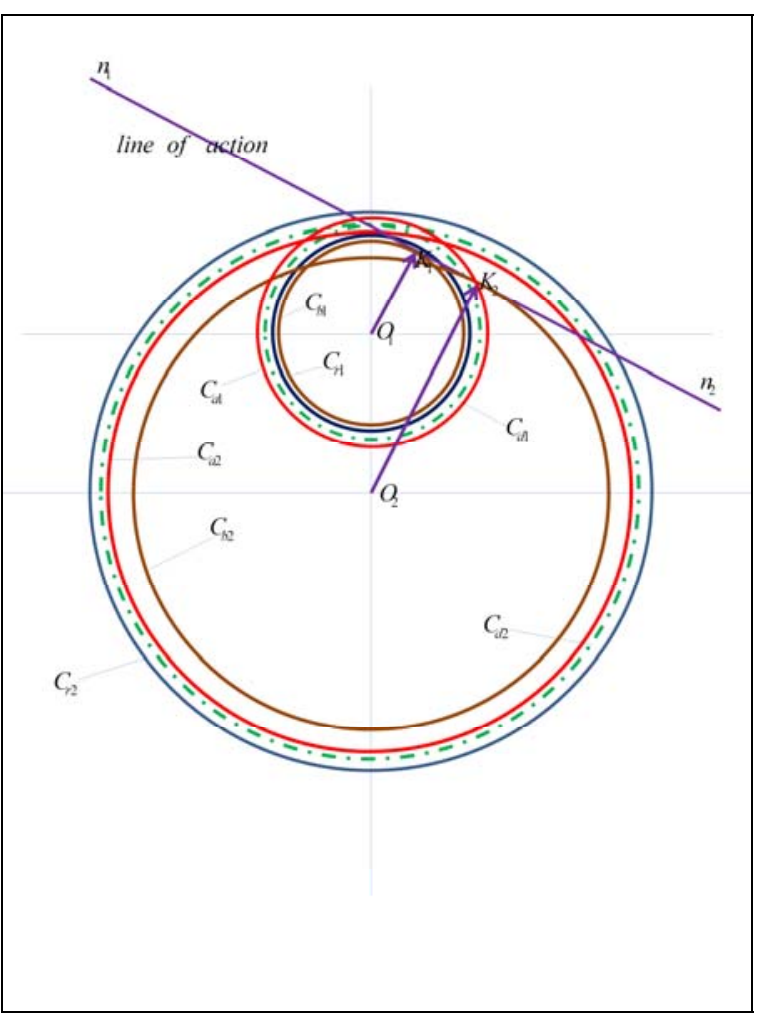

Figure 9. An internal gearing

To an external gearing contact between profiles shall only be made to a single point, while at the internal gearing the contact between profiles is by winding each other (see the Figure 10 and (PETRESCU, 2012)).

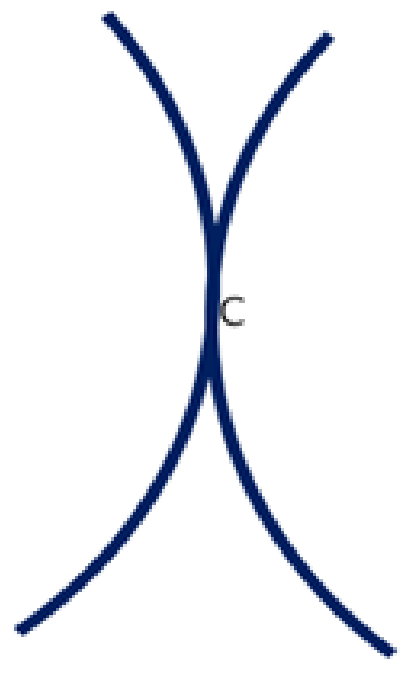

External gearing

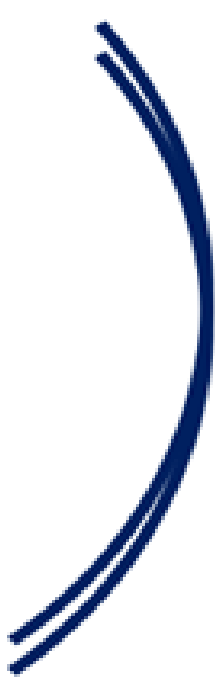

Internal gearing

Figure 10. Contact between profiles 


\section{GEARS SYNTHESIS BY AVOID THE INTERFERENCES}

In order to avoid interference phenomenon, point $\mathrm{A}$ must lie between $\mathrm{C}$ and $\mathrm{K}_{1}$ (the addendum circle of the wheel $2, \mathrm{C}_{\mathrm{a} 2}$ need to cut the line of action between points $\mathrm{C}$ and $\mathrm{K}_{1}$, and under no circumstances does not exceed the point $\mathrm{K}_{1}$ ). Similarly, $\mathrm{C}_{\mathrm{a} 1}$ addendum circle must cut the action line between points $C$ and $K_{2}$, resulting point $E$, which in no circumstances, does not exceed the point $\mathrm{K}_{2}$.

The conditions to avoid the phenomenon of interference can be written with the relations (3.1).

The basic conditions of interference, are the same $\left(\mathrm{CA}<\mathrm{K}_{1} \mathrm{C} ; \mathrm{CE}<\mathrm{K}_{2} \mathrm{C}\right)$, but the originality of this new presented method consist in the mode in which it was solved the classical relationship (see the system 3.1) (PETRESCU, 2012), (see the Figure 11). 


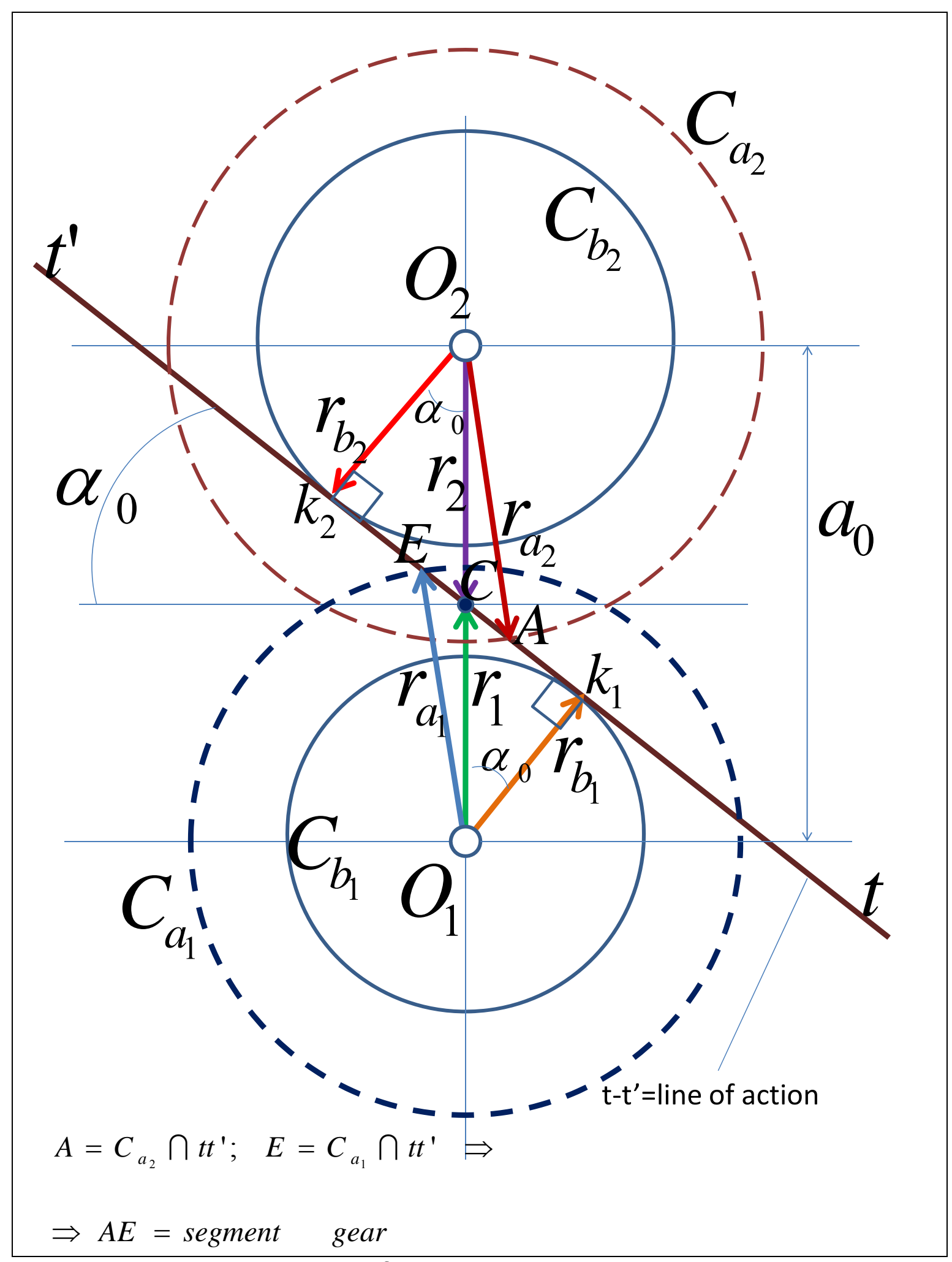

Figure 11. Line of action (t-t') at an external gearing 


$$
\begin{aligned}
& C A<K_{1} C \text { and } C E<K_{2} C \\
& C A=K_{2} A-K_{2} C=\sqrt{r_{a_{2}}^{2}-r_{b_{2}}^{2}}-r_{2} \cdot \sin \alpha_{0} ; \quad C A<K_{1} C \Rightarrow \\
& \Rightarrow \sqrt{r_{a_{2}}^{2}-r_{b_{2}}^{2}}-r_{2} \cdot \sin \alpha_{0}<r_{1} \cdot \sin \alpha_{0} \Rightarrow \sqrt{r_{a_{2}}^{2}-r_{b_{2}}^{2}}<\left(r_{1}+r_{2}\right) \cdot \sin \alpha_{0} \\
& \Rightarrow d_{a_{2}}^{2}-d_{b_{2}}^{2}<\left(d_{1}+d_{2}\right)^{2} \cdot \sin ^{2} \alpha_{0} \Rightarrow \\
& \Rightarrow m^{2} \cdot\left(z_{2}+2\right)^{2}-m^{2} \cdot z_{2}^{2} \cdot \cos ^{2} \alpha_{0}<m^{2} \cdot\left(z_{1}+z_{2}\right)^{2} \cdot \sin ^{2} \alpha_{0} \Rightarrow \\
& \Rightarrow z_{2}^{2}+4 \cdot z_{2}+4-z_{2}^{2}<z_{1}^{2} \cdot \sin ^{2} \alpha_{0}+2 \cdot z_{1} \cdot z_{2} \cdot \sin ^{2} \alpha_{0} \Rightarrow \\
& \Rightarrow 4 \cdot z_{2}+4<z_{1}^{2} \cdot \sin ^{2} \alpha_{0}+2 \cdot z_{1} \cdot z_{2} \cdot \sin ^{2} \alpha_{0} \\
& \text { from } C E<K_{2} C \Rightarrow 4 \cdot z_{1}+4<z_{2}^{2} \cdot \sin ^{2} \alpha_{0}+2 \cdot z_{1} \cdot z_{2} \cdot \sin ^{2} \alpha_{0} \\
& \text { it obtains the system }\left\{\begin{array}{l}
4 \cdot z_{2}+4<z_{1}^{2} \cdot \sin ^{2} \alpha_{0}+2 \cdot z_{1} \cdot z_{2} \cdot \sin ^{2} \alpha_{0} \\
4 \cdot z_{1}+4<z_{2}^{2} \cdot \sin ^{2} \alpha_{0}+2 \cdot z_{1} \cdot z_{2} \cdot \sin ^{2} \alpha_{0}
\end{array}\right. \\
& \text { take } i \equiv\left|i_{12}\right|=\frac{z_{2}}{z_{1}} \Rightarrow z_{2}=i \cdot z_{1} \text {; result the system } \\
& \left\{\begin{array}{l}
\sin ^{2} \alpha_{0} \cdot(1+2 \cdot i) \cdot z_{1}^{2}-2 \cdot 2 \cdot i \cdot z_{1}-4>0 \\
\sin ^{2} \alpha_{0} \cdot\left(i^{2}+2 \cdot i\right) \cdot z_{1}^{2}-2 \cdot 2 \cdot z_{1}-4>0
\end{array}\right. \text { with the solutions: } \\
& z_{1_{1,2}}=\frac{2 \cdot i \pm 2 \cdot \sqrt{i^{2}+\sin ^{2} \alpha_{0}+2 \cdot i \cdot \sin ^{2} \alpha_{0}}}{(2 \cdot i+1) \cdot \sin ^{2} \alpha_{0}} \\
& \left\{z_{1_{3,4}}=\frac{2 \pm 2 \cdot \sqrt{1+i^{2} \cdot \sin ^{2} \alpha_{0}+2 \cdot i \cdot \sin ^{2} \alpha_{0}}}{\left(2 \cdot i+i^{2}\right) \cdot \sin ^{2} \alpha_{0}} \text { it keeps solutions }+\right. \\
& \left\{\begin{array}{l}
z_{1_{2}}=2 \cdot \frac{i+\sqrt{i^{2}+\sin ^{2} \alpha_{0}+2 \cdot i \cdot \sin ^{2} \alpha_{0}}}{(2 \cdot i+1) \cdot \sin ^{2} \alpha_{0}} \\
z_{1_{4}}=2 \cdot \frac{1+\sqrt{1+i^{2} \cdot \sin ^{2} \alpha_{0}+2 \cdot i \cdot \sin ^{2} \alpha_{0}}}{\left(2 \cdot i+i^{2}\right) \cdot \sin ^{2} \alpha_{0}}
\end{array}\right.
\end{aligned}
$$

Relationship which generates $z_{1_{4}}$ always gives lower values than the relationship which generates $z_{1_{2}}$ so it is sufficient the condition (3.2) for finding the minimum number of teeth of the wheel 1 , necessary to avoid interference (PETRESCU, 2012).

$$
z_{\text {min }} \equiv z_{1_{2}}=2 \cdot \frac{i+\sqrt{i^{2}+\sin ^{2} \alpha_{0}+2 \cdot i \cdot \sin ^{2} \alpha_{0}}}{(2 \cdot i+1) \cdot \sin ^{2} \alpha_{0}}
$$


When we have inclined teeth, one takes $z_{\min } \rightarrow z_{\min } / \cos \beta$, and $\alpha_{0} \rightarrow \alpha_{0 t}$, and the relationship (3.2) takes the form (3.3). The minimum number of teeth of the driving wheel 1 , is a function on some parameters: the pressure angle (normal on the pitch circle, $\left.\alpha_{0}\right)$, the tooth inclination angle $(\beta)$, and the transmission ratio $\left(i=\left|i_{12}\right|=\mid-\right.$ $\mathrm{z}_{2} / \mathrm{z}_{1} \mid=\mathrm{z}_{2} / \mathrm{z}_{1}$ ), (see the relationship 3.3, and (MAROŞ, 1958); STOICA, 1977).

$$
\left\{\begin{array}{l}
z_{\text {min }} \equiv z_{1_{2}}=2 \cdot \cos \beta \cdot \frac{i+\sqrt{i^{2}+\sin ^{2} \alpha_{0 t}+2 \cdot i \cdot \sin ^{2} \alpha_{0 t}}}{(2 \cdot i+1) \cdot \sin ^{2} \alpha_{0 t}} \\
\text { where }: \operatorname{tg} \alpha_{0 t}=\frac{\operatorname{tg} \alpha_{0}}{\cos \beta} \Rightarrow \\
\Rightarrow \alpha_{0 t}=\operatorname{arctg}\left(\frac{\operatorname{tg} \alpha_{0}}{\cos \beta}\right)
\end{array}\right.
$$

The system (3.3) is a simple, unitar and general relationship which can give the solutions of the minimum number of teeth of the wheel 1 (the driving wheel), to avoid the interference. In the following tables (1-15) is chosen an alpha0 value (35 [deg]), and successively increased beta angle values (from 0 [deg] to 40 [deg]) and the transmission ratio $\mathrm{i}$ (from 1, to 80 ), and one gets the minimum numbers of teeth. Then, we will decrease successively the value of the angle alpha0 (from 35 [deg] to 5 [deg]). See the tables (1-15).

At the internal gearing the condition to avoid the interference is the same like at the external gearing (relation 3.3).

In addition it can write and the condition of the existence of the wheel with the internal teeth (systems 3.4 and 3.5).

$$
\left\{\begin{array}{l}
r_{a_{2}}>r_{b_{2}} \Rightarrow \frac{m}{2} \cdot\left(\frac{z_{2}}{\cos \beta}-2\right)>\frac{m}{2} \cdot \frac{z_{2}}{\cos \beta} \cdot \cos \alpha_{0 t} \Rightarrow \\
\Rightarrow \frac{z_{2}}{\cos \beta}-2>\frac{z_{2}}{\cos \beta} \cdot \cos \alpha_{0 t} \Rightarrow z_{2}>\frac{2 \cdot \cos \beta}{1-\cos \alpha_{0 t}}
\end{array}\right.
$$




$$
\left\{\begin{array}{l}
z_{2}>\frac{2 \cdot \cos \beta}{1-\cos \alpha_{0 t}} \\
\cos \alpha_{0 t}=\frac{1}{\sqrt{1+\operatorname{tg}^{2} \alpha_{0 t}}}=\frac{1}{\sqrt{1+\frac{\operatorname{tg}^{2} \alpha_{0}}{\cos ^{2} \beta}}}=\frac{\cos \beta}{\sqrt{\cos ^{2} \beta+\operatorname{tg}^{2} \alpha_{0}}} \\
\Rightarrow z_{2}>\frac{2 \cdot \cos \beta}{1-\frac{\cos \beta}{\sqrt{\cos ^{2} \beta+\operatorname{tg}^{2} \alpha_{0}}} \Rightarrow z_{2}}>\frac{2 \cdot \cos \beta \cdot \sqrt{\cos ^{2} \beta+\operatorname{tg}^{2} \alpha_{0}}}{\sqrt{\cos ^{2} \beta+\operatorname{tg}^{2} \alpha_{0}}-\cos \beta} \Rightarrow \\
\Rightarrow z_{2}>\frac{2 \cdot \cos \beta \cdot \sqrt{\cos ^{2} \beta+\operatorname{tg}^{2} \alpha_{0}} \cdot\left(\sqrt{\cos ^{2} \beta+\operatorname{tg}^{2} \alpha_{0}}+\cos \beta\right)}{\operatorname{tg}^{2} \alpha_{0}} \Rightarrow \\
\Rightarrow z_{2}>\frac{2 \cdot \cos ^{2} \beta}{\operatorname{tg}^{2} \alpha_{0}} \cdot\left(\cos ^{2} \beta+\operatorname{tg}^{2} \alpha_{0}+\cos \beta \cdot \sqrt{\cos ^{2} \beta+\operatorname{tg}^{2} \alpha_{0}}\right) \Rightarrow \\
\Rightarrow z_{2}>\frac{2 \cdot \cos ^{3} \beta}{\operatorname{tg}^{2} \alpha_{0}} \cdot\left(1+\frac{\operatorname{tg}^{2} \alpha_{0}}{\cos ^{2} \beta}+\sqrt{1+\frac{\operatorname{tg}^{2} \alpha_{0}}{\cos ^{2} \beta}}\right)
\end{array}\right.
$$

They were used and additional relations (3.6).

$$
\left\{\begin{array}{l}
r_{1}=\frac{1}{2} m \cdot z_{1} ; \quad r_{2}=\frac{1}{2} m \cdot z_{2} ; \quad r_{b_{1}}=\frac{1}{2} m \cdot z_{1} \cdot \cos \alpha_{0} ; r_{b_{2}}=\frac{1}{2} m \cdot z_{2} \cdot \cos \alpha_{0} \\
r_{a_{1}}=r_{1}+m=\frac{1}{2} m \cdot z_{1}+\frac{2}{2} m=\frac{m}{2} \cdot\left(z_{1}+2\right) \\
r_{a_{2}}=r_{2}-m=\frac{1}{2} m \cdot z_{2}-\frac{2}{2} m=\frac{m}{2} \cdot\left(z_{2}-2\right) \\
r_{r_{1}}=r_{1}-1.25 m=\frac{1}{2} m \cdot z_{1}-\frac{2.5}{2} m=\frac{m}{2} \cdot\left(z_{1}-2.5\right) \\
r_{r_{2}}=r_{2}+1.25 m=\frac{1}{2} m \cdot z_{2}+\frac{2.5}{2} m=\frac{m}{2} \cdot\left(z_{2}+2.5\right)
\end{array}\right.
$$

Table $1 \alpha 0=35$ [deg], $\beta=0$ [deg] 
INDEPENDENT JOURNAL OF MANAGEMENT \& PRODUCTION (IJM\&P) http://www.ijmp.jor.br

v. 5, n. 2, February - May 2014. ISSN: 2236-269X

DOI: 10.14807/ijmp.v5i2.147

\begin{tabular}{c|ccccccccccc}
$\begin{array}{c}\alpha_{0} \\
{[\text { deg] }}\end{array}$ & 35 & & $\beta$ [deg] & 0 \\
$\mathrm{i}$ & 1 & 1.25 & 1.6 & 2 & 2.5 & 3.15 & 4 & 5 & 6.3 & 8 \\
\hline $\mathrm{Z}_{\min }$ & 4.8828 & 5.0325 & 5.1896 & 5.3204 & 5.4386 & 5.5467 & 5.6431 & 5.7198 & 5.7867 & 5.8439 \\
& & & & & & & & & & & \\
$\mathrm{i}$ & 10 & 12.5 & 16 & 20 & 25 & 31.5 & 40 & 50 & 63 & 80 \\
$\mathrm{Z}_{\min }$ & 5.88880 & 5.9243 & 5.9568 & 5.9805 & 5.9997 & 6.0158 & 6.0290 & 6.0389 & 6.0471 & 6.0539 \\
\hline
\end{tabular}

Table $2 \alpha_{0}=35$ [deg], $\beta=10$ [deg]

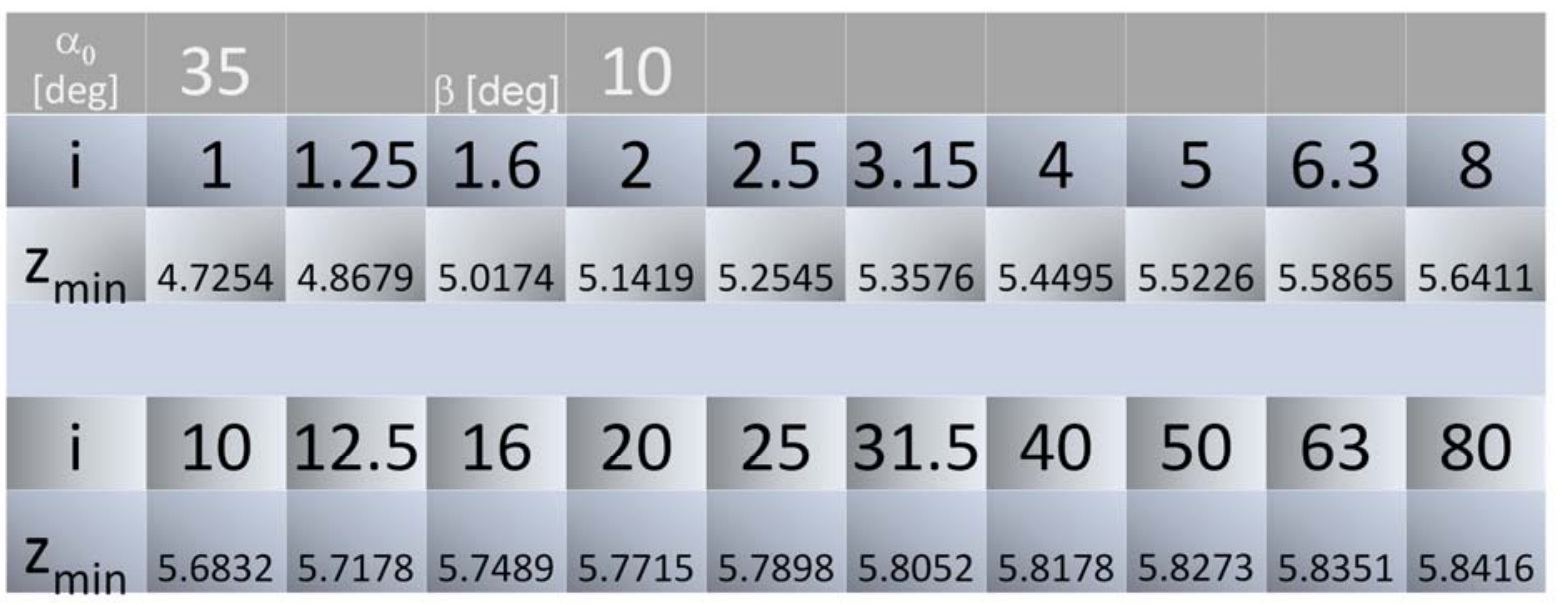

Table $3 \alpha 0=35$ [deg], $\beta=20$ [deg]

\begin{tabular}{|c|c|c|c|c|c|}
\hline $\left.\begin{array}{c}\alpha_{0} \\
{[\operatorname{deg}]}\end{array}\right] 35$ & 20 & & & & \\
\hline $\begin{array}{llll}\text { i } & 1 & 1.25 & 1.6\end{array}$ & 2 & $2.53 .15 \quad 4$ & 5 & & 8 \\
\hline$Z_{\min } 4.27994 .40224 .5307$ & 4.6379 & 4.73514 .82404 .903 & 1.96 & 5.0219 & 5.0 \\
\hline $\begin{array}{lll}10 & 12.5 & 16\end{array}$ & 20 & $\begin{array}{lll}25 & 31.5 & 40\end{array}$ & 50 & 63 & 80 \\
\hline
\end{tabular}


INDEPENDENT JOURNAL OF MANAGEMENT \& PRODUCTION (IJM\&P)

http://www.ijmp.jor.br

v. 5, n. 2, February - May 2014.

ISSN: 2236-269X

DOI: 10.14807/ijmp.v5i2.147

Table $4 \alpha 0=35$ [deg], $\beta=30$ [deg]

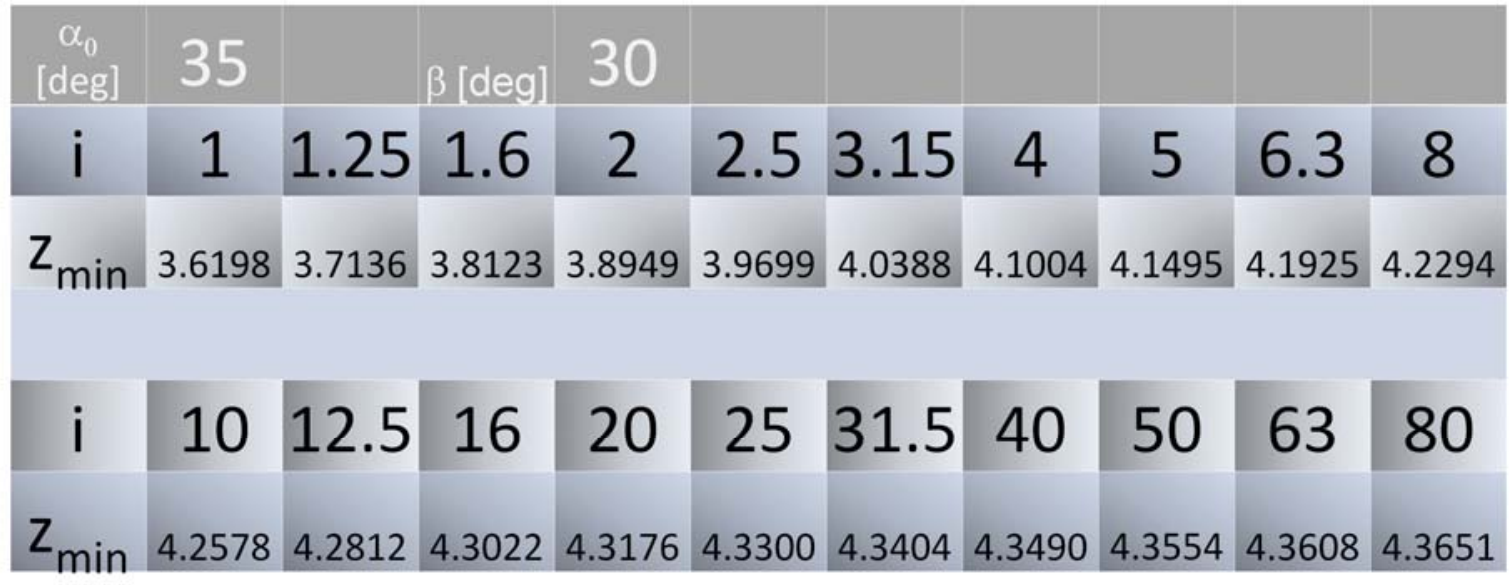

Table $5 \alpha 0=35$ [deg], $\beta=40$ [deg]

\begin{tabular}{|c|c|c|c|c|c|c|c|c|c|c|}
$\begin{array}{c}\alpha_{0} \\
{[\text { deg] }}\end{array}$ & 35 & & $\beta$ [deg] & 40 \\
$\mathrm{i}$ & 1 & 1.25 & 1.6 & 2 & 2.5 & 3.15 & 4 & 5 & 6.3 & 8 \\
\hline $\mathrm{z}_{\min }$ & 2.8475 & 2.9104 & 2.9769 & 3.0327 & 3.0836 & 3.1304 & 3.1724 & 3.2060 & 3.2355 & 3.2608 \\
$\mathrm{i}$ & 10 & 12.5 & 16 & 20 & 25 & 31.5 & 40 & 50 & 63 & 80 \\
$\mathrm{z}_{\min }$ & 3.2804 & 3.2965 & 3.3110 & 3.3216 & 3.3302 & 3.3374 & 3.3433 & 3.3477 & 3.3514 & 3.3545 \\
\hline
\end{tabular}

Table $6 \alpha 0=20$ [deg], $\beta=0$ [deg]

\begin{tabular}{|c|c|c|c|c|c|c|c|c|c|c|}
\hline $\begin{array}{c}\alpha_{0} \\
{[\text { deg] }}\end{array}$ & 20 & & $\beta$ [deg] & 0 & & & & & \\
\hline $\mathrm{i}$ & 1 & 1.25 & 1.6 & 2 & 2.5 & 3.15 & 4 & 5 & 6.3 & 8 \\
\hline $\mathrm{z}_{\min }$ & 12.323 & 12.966 & 13.624 & 14.161 & 14.637 & 15.066 & 15.443 & 15.740 & 15.997 & 16.215 \\
\hline $\mathrm{i}$ & 10 & 12.5 & 16 & 20 & 25 & 31.5 & 40 & 50 & 63 & 80 \\
$\mathrm{z}_{\min }$ & 16.382 & 16.519 & 16.641 & 16.730 & 16.802 & 16.862 & 16.911 & 16.948 & 16.978 & 17.003 \\
\hline
\end{tabular}


INDEPENDENT JOURNAL OF MANAGEMENT \& PRODUCTION (IJM\&P)

http://www.ijmp.jor.br

v. 5, n. 2, February - May 2014.

ISSN: 2236-269X

DOI: 10.14807/ijmp.v5i2.147

Table $7 \alpha 0=20$ [deg], $\beta=10$ [deg]

\begin{tabular}{|c|c|c|c|c|c|c|c|c|c|c|}
\hline $\begin{array}{c}\alpha_{0} \\
\text { [deg] }\end{array}$ & 20 & & $\beta$ [deg] & 10 & & & & & & \\
\hline i & 1 & 1.25 & 1.6 & 2 & 2.5 & 3.15 & 4 & 5 & 6.3 & 8 \\
\hline $\mathrm{Z}_{\text {min }}$ & 11.835 & 12.447 & 13.075 & 13.586 & 14.041 & 14.450 & 14.810 & 15.094 & 15.339 & 15.547 \\
\hline i & 10 & 12.5 & 16 & 20 & 25 & 31.5 & 40 & 50 & 63 & 80 \\
\hline$z_{\min }$ & 15.706 & 15.837 & 15.954 & 16.039 & 16.107 & 16.164 & 16.211 & 16.246 & 16.275 & 16.299 \\
\hline
\end{tabular}

Table $8 \alpha 0=20$ [deg], $\beta=20$ [deg]

\begin{tabular}{|c|c|c|c|c|c|c|c|c|c|c|c|c|}
\hline $\begin{array}{c}\alpha_{0} \\
{[\mathrm{deg}]}\end{array}$ & 20 & \multicolumn{1}{c}{$\beta$ [deg] } & 20 \\
\hline $\mathrm{i}$ & 1 & 1.25 & 1.6 & 2 & 2.5 & 3.15 & 4 & 5 & 6.3 & 8 \\
\hline $\mathrm{z}_{\min }$ & 10.446 & 10.994 & 11.535 & 11.977 & 12.370 & 12.725 & 13.036 & 13.282 & 13.495 & 13.676 \\
\hline $\mathbf{i}$ & 10 & 12.5 & 16 & 20 & 25 & 31.5 & 40 & 50 & 63 & 80 \\
\hline $\mathrm{Z}_{\min }$ & 13.814 & 13.927 & 14.028 & 14.102 & 14.161 & 14.211 & 14.252 & 14.282 & 14.308 & 14.328 \\
\hline
\end{tabular}

Table $9 \alpha 0=20$ [deg], $\beta=30$ [deg]

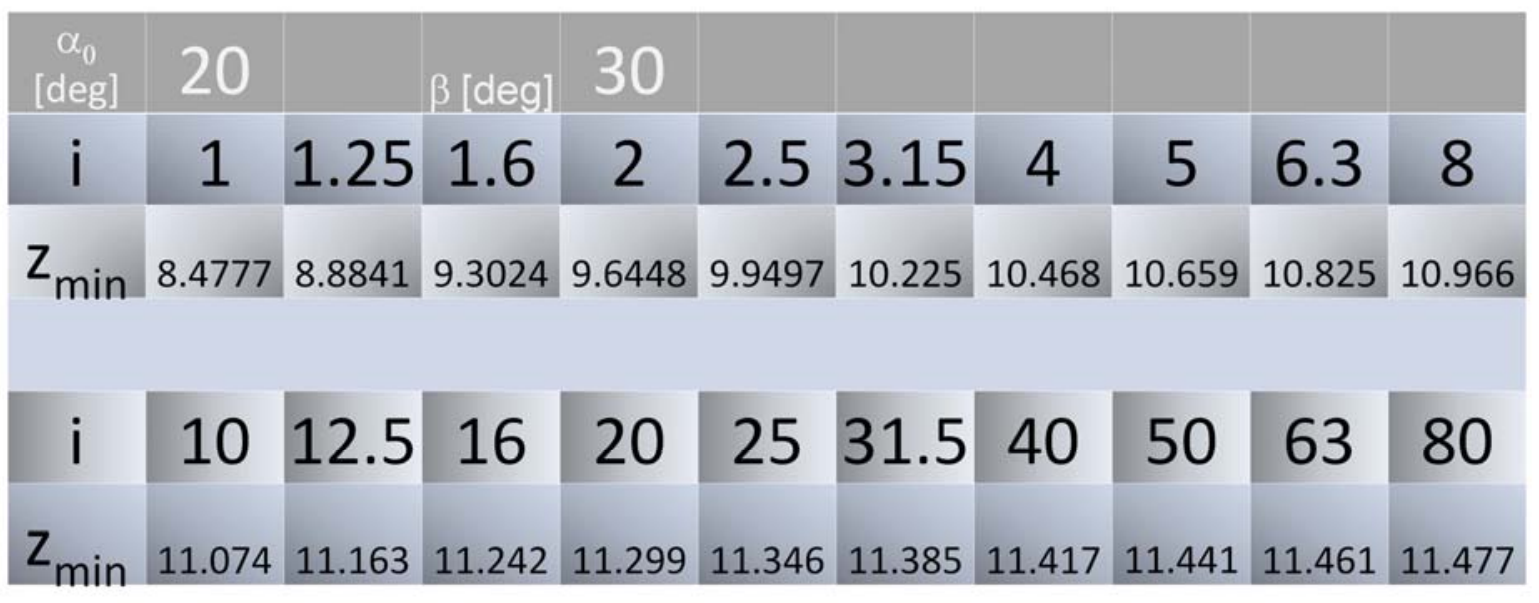


INDEPENDENT JOURNAL OF MANAGEMENT \& PRODUCTION (IJM\&P) http://www.ijmp.jor.br

v. 5, n. 2, February - May 2014. ISSN: 2236-269X

DOI: 10.14807/ijmp.v5i2.147

Table $10 \alpha 0=20$ [deg], $\beta=40$ [deg]

\begin{tabular}{c|c|c|c|c|c|c|c|c|c|c|}
\hline $\begin{array}{c}\alpha_{0} \\
{[\text { deg] }}\end{array}$ & 20 & & $\beta$ [deg] & 40 & & & & & \\
\hline $\mathrm{i}$ & 1 & 1.25 & 1.6 & 2 & 2.5 & 3.15 & 4 & 5 & 6.3 & 8 \\
\hline $\mathrm{z}_{\min }$ & 6.2280 & 6.5020 & 6.7854 & 7.0182 & 7.2263 & 7.4147 & 7.5813 & 7.7128 & 7.8269 & 7.9241 \\
\hline $\mathrm{i}$ & 10 & 12.5 & 16 & 20 & 25 & 31.5 & 40 & 50 & 63 & 80 \\
\hline $\mathrm{z}_{\min }$ & 7.9985 & 8.0597 & 8.1143 & 8.1540 & 8.1862 & 8.2131 & 8.2352 & 8.2517 & 8.2654 & 8.2767 \\
\hline
\end{tabular}

Table $11 \alpha 0=5$ [deg], $\beta=0$ [deg]

\begin{tabular}{|c|c|c|c|c|c|c|c|c|c|c|}
\hline $\begin{array}{c}\alpha_{0} \\
{[\mathrm{deg}]}\end{array}$ & 5 & & $\beta$ [deg] & 0 & & & & & & \\
\hline i & 1 & 1.25 & 1.6 & 2 & 2.5 & 3.15 & 4 & 5 & 6.3 & 8 \\
\hline$z_{\min }$ & 176.52 & 188.86 & 201.22 & 211.13 & 219.81 & 227.54 & 234.28 & 239.55 & 244.09 & 247.93 \\
\hline i & 10 & 12.5 & 16 & 20 & 25 & 31.5 & 40 & 50 & 63 & 80 \\
\hline
\end{tabular}

Table $12 \alpha 0=5$ [deg], $\beta=10$ [deg]

\begin{tabular}{|c|c|c|c|c|c|c|c|c|c|c|}
\hline $\begin{array}{c}\alpha_{0} \\
\text { [deg] }\end{array}$ & 5 & & $\beta$ [deg] & 10 & & & & & \\
\hline $\mathrm{i}$ & 1 & 1.25 & 1.6 & 2 & 2.5 & 3.15 & 4 & 5 & 6.3 & 8 \\
\hline $\mathrm{z}_{\min }$ & 168.66 & 180.45 & 192.25 & 201.72 & 210.01 & 217.38 & 223.83 & 228.86 & 233.19 & 236.86 \\
\hline $\mathrm{i}$ & 10 & 12.5 & 16 & 20 & 25 & 31.5 & 40 & 50 & 63 & 80 \\
\hline $\mathrm{z}_{\min }$ & 239.65 & 241.94 & 243.97 & 245.45 & 246.64 & 247.63 & 248.45 & 249.06 & 249.57 & 249.98 \\
\hline
\end{tabular}


INDEPENDENT JOURNAL OF MANAGEMENT \& PRODUCTION (IJM\&P) http://www.ijmp.jor.br

v. 5, n. 2, February - May 2014. ISSN: 2236-269X

DOI: 10.14807/ijmp.v5i2.147

Table $13 \alpha 0=5$ [deg], $\beta=20$ [deg]

\begin{tabular}{c|ccc|c|c|c|c|c|c|c}
$\begin{array}{c}\alpha_{0} \\
{[\text { deg] }}\end{array}$ & 5 & & $\beta$ [deg] & 20 \\
$\mathrm{i}$ & 1 & 1.25 & 1.6 & 2 & 2.5 & 3.15 & 4 & 5 & 6.3 & 8 \\
$\mathrm{Z}_{\min }$ & 146.73 & 156.95 & 167.20 & 175.42 & 182.62 & 189.03 & 194.63 & 198.99 & 202.76 & 205.94 \\
$\mathrm{i}$ & 10 & 12.5 & 16 & 20 & 25 & 31.5 & 40 & 50 & 63 & 80 \\
$\mathrm{Z}_{\min }$ & 208.37 & 210.35 & 212.12 & 213.40 & 214.44 & 215.30 & 216.01 & 216.54 & 216.98 & 217.34
\end{tabular}

Table $14 \alpha 0=5$ [deg], $\beta=30$ [deg]

\begin{tabular}{c|ccccccccccc}
$\begin{array}{c}\alpha_{0} \\
{[\text { deg] }}\end{array}$ & 5 & \multicolumn{1}{c}{$\beta$ [deg] } & 30 \\
$\mathrm{i}$ & 1 & 1.25 & 1.6 & 2 & 2.5 & 3.15 & 4 & 5 & 6.3 & 8 \\
$\mathrm{z}_{\min }$ & 115.16 & 123.15 & 131.16 & 137.58 & 143.22 & 148.23 & 152.61 & 156.03 & 158.97 & 161.47 \\
$\mathrm{i}$ & 10 & 12.5 & 16 & 20 & 25 & 31.5 & 40 & 50 & 63 & 80 \\
$\mathrm{z}_{\min }$ & 163.37 & 164.92 & 166.30 & 167.31 & 168.12 & 168.79 & 169.35 & 169.76 & 170.11 & 170.39 \\
\hline
\end{tabular}

Table $15 \alpha 0=5$ [deg], $\beta=40$ [deg]

\begin{tabular}{c|c|c|c|c|c|c|c|c|c|c|c|}
\hline $\begin{array}{c}\alpha_{0} \\
{[\text { deg] }}\end{array}$ & 5 & & $\beta$ [deg] & 40 & & & & & & \\
\hline $\mathrm{i}$ & 1 & 1.25 & 1.6 & 2 & 2.5 & 3.15 & 4 & 5 & 6.3 & 8 \\
\hline $\mathrm{z}_{\min }$ & 80.086 & 85.602 & 91.136 & 95.575 & 99.465 & 102.93 & 105.96 & 108.33 & 110.36 & 112.09 \\
\hline $\mathrm{i}$ & 10 & 12.5 & 16 & 20 & 25 & 31.5 & 40 & 50 & 63 & 80 \\
\hline $\mathrm{z}_{\min }$ & 113.40 & 114.48 & 115.43 & 116.13 & 116.69 & 117.16 & 117.54 & 117.83 & 118.07 & 118.26 \\
\hline
\end{tabular}


INDEPENDENT JOURNAL OF MANAGEMENT \& PRODUCTION (IJM\&P)

http://www.ijmp.jor.br

v. 5, n. 2, February - May 2014.

ISSN: 2236-269X

DOI: 10.14807/ijmp.v5i2.147

The presented method has the great advantage to optimize the number of teeth for a gear before to make its synthesis. In this mode the constructor may elect the minimum number of teeth, for an imposed transmission ratio, i.

Classical to realize an $\mathrm{i}=2$, the constructor can select between 18 or 33 teeth to the driving wheel 1 , which means a 36 or 66 teeth for the driven wheel 2 . With the aid of the presented tables, he can make a multiple selection.

The engineer can select for the driving wheel 1 a number of teeth $z_{1}=6$, with an alpha $_{0}=35$ [deg], and a beta=0 [deg]. He may do this not only for a transmission ratio $\mathrm{i}=2$, but and for the domain from $\mathrm{i}=1.25$ to $\mathrm{i}=25$ (see the table 1 ).

If he elect alpha $a_{0}=35$ [deg] and beta=40 [deg], then he can take a number of teeth for the driving wheel 1 of $z_{1}=4$, and can do this for the entire domain from $i=2$ to $i=80$ (see the table 5). The constructor may do this when it is necessary a minimum number of teeth, but with an efficiency of the gear decreasing.

Contrary, when we wish a great efficiency, one must increase the number of teeth and decrease the angles alpha $a_{0}$ and beta. With an alpha $a_{0}=5$ [deg] and beta $=0$ (table 11), we can take the number of teeth at the driving wheel 1 , from $z_{1}=189$ (for $\mathrm{i}=1.25$ ), to $\mathrm{z}_{1}=262$ (for $\mathrm{i}=80$ ).

For the known classical alpha $a_{0}=20$ [deg] and beta=0 [deg], when the ratio $\mathrm{i}$ vary from 1 to $80, z_{1}$ takes the values from 13 to 18 (see the table 6 ). With the classical method it was taken only the minimum value 18 to the minimum number of teeth (PETRESCU, 2012).

\section{CONCLUSIONS}

The presented method manages to synthesize (in theory) the best option parameters for any desired gear.

Relationships shown have the great advantage of donating optimal solutions for any situation you want without the need for difficult calculations, experimental building, or specialized software. All relationships have been calculated and checked with programs written in excel.

Comparisons made with specialized software (Inventor) showed a precision (matching) perfect. Workload and procedures could be so much smaller. 
INDEPENDENT JOURNAL OF MANAGEMENT \& PRODUCTION (IJM\&P)

http://www.ijmp.jor.br

v. 5, n. 2, February - May 2014.

ISSN: 2236-269X

DOI: 10.14807/ijmp.v5i2.147

The parallel drawn between the software "Autodesk Inventor" and the presented calculation relationships will be highlighted in the following paper (as handle a large volume), (OLIVEIRA; LIMA, 2003).

Applied in the automotive industry, at the transmission mechanisms, these changes may decrease overall fuel consumption further, and pollutant emissions (NOGUEIRA; REAL, 2011).

Gears can be designed to operate without noise (PELECUDI et al., 1985), (PETRESCU; PETRESCU; POPESCU, 2007; MAROŞ, 1958), (LENI MATOS, 2011; NOGUEIRA; REAL, 2011).

But, the applications will be spectacular in the automatic transmissions used in aerospace, in robotics and mechatronics.

When the number of teeth of the wheel 1 increases, it can decrease the normal pressure angle, $\alpha_{0}$. One shall see that for $z_{1}=90$ it can take less for the normal pressure angle (for the pressure angle of reference), $\alpha_{0}=8^{0}$.

The efficiency (of the gear) increases when the number of teeth for the driving wheel $1, z_{1}$, increases as well, and when the pressure angle, $a_{0}$, diminishes; $z_{2}$ or $i_{12}$ are not so much influence about the efficiency value;

It can easily see that for the value $\alpha_{0}=20^{\circ}$, the efficiency takes roughly the value $\eta \approx 0.89$ for any values of the others parameters (this justifies the choice of this value, $\alpha_{0}=20^{\circ}$, for the standard pressure angle of reference).

The better efficiency may be obtained only for a $\alpha_{0} \neq 20^{0}$.

But the pressure angle of reference, $\alpha_{0}$, can be decreased the same time the number of teeth for the driving wheel $1, z_{1}$, increases, to increase the gears' efficiency;

Contrary, when we desire to create a gear with a low $z_{1}$ (for a less gauge), it will be necessary to increase the $\alpha_{0}$ value, for maintaining a positive value for $\alpha_{m}$ (in this case the gear efficiency will be diminished);

When $\beta$ increases, the efficiency, $\eta$, increases too, but the growth is insignificant.

The module of the gear, $m$, has not any influence on the gear's efficiency value.

When $\alpha_{0}$ is diminished it can take a higher normal module, for increasing the addendum of teeth, but the increase of the module $\mathrm{m}$ at the same time with the increase of the $z_{1}$ can lead to a greater gauge. 
The gears' efficiency, $\eta$, is really a function of $\alpha_{0}$ and $z_{1}: \eta=f\left(\alpha_{0}, z_{1}\right) ; \alpha_{m}$ and $\alpha_{M}$ are just the intermediate parameters.

For a good projection of the gear, it's necessary a z1 and a z2 greater than 3060 ; but this condition may increase the gauge of mechanism.

The best efficiency can be obtained with the internal gearing when the drive wheel 1 is the ring; the minimum efficiency will be obtained when the drive wheel 1 of the internal gearing has external teeth.

For the external gearing, the best efficiency is obtained when the bigger wheel is the drive wheel; when we decrease the normal angle $\alpha 0$, the contact ratio increases and the efficiency increases as well.

The efficiency increases too, when the number of teeth of the drive wheel 1 increases (when z1 increases).

Optimizing this mechanism (the gears mechanism), we can improve the functionality of the transmissions with gears.

At the gear mechanisms an important problem is the interference of the teeth. To avoid the interference between teeth, we must know the minimum number of teeth of the driving wheel, in function of the pressure angle (normal on the pitch circle, alpha0), in function of the tooth inclination angle (beta), and in function of the transmission ratio (i).

\section{REFERENCES}

LEI X., A. O. (2011), Design and Analysis for High-speed Gear Coupling, Applied Mechanics and Materials journal, v. 86, p. 658-661.

LIN C., A. O. (2011), The Design and Experiments of Oval Bevel Gear, Applied Mechanics and Materials journal, v. 86, p. 297-300.

MAROŞ, D. (1958), Cinematica roţilor dinţate. Editura Tehnică, Bucureşti.

LENI MATOS, B. M. (2011), A Comparison of the Delay Spread Obtained with Different Power Delay Profiles De-Noising Techniques, ENGEVISTA, v. 13, n. 2, p. 129-133. Available on:

http://www.uff.br/engevista/seer/index.php/engevista/article/view/280

NOGUEIRA, O. C.; REAL, M. V. (2011), Estudo Comparativo De Motores Diesel Maritimos Atraves Da Analise De Lubrificantes Usados e Engehharia De Confiabilidade, ENGEVISTA, v. 13, n. 3, p. 244-254. Available on: http://www.uff.br/engevista/seer/index.php/engevista/article/view/300 
OLIVEIRA, M. M. P.; LIMA, F. R. (2003), Analogias Entre o Desenho Instrumental e o Dedenho Computacional, in ENGEVISTA, v.5, n. 2, Available on:

http://www.uff.br/engevista/seer/index.php/engevista/article/view/108

PELECUDI, C. H. R.; MAROS, D.; MERTICARU, V.; PANDREA, N.; SIMIONESCU, I. (1985), Mecanisme. E.D.P., Bucureşti.

PETRESCU, V.; PETRESCU, I. (2002), Randamentul cuplei superioare de la angrenajele cu roţi dinţate cu axe fixe, In: The Proceedings of $7^{\text {th }}$ National Symposium PRASIC, Braşov, v. I, p. 333-338.

PETRESCU, R.; PETRESCU, F. (2003), The gear synthesis with the best efficiency, In: The Proceedings of ESFA'03, Bucharest, v. 2, p. 63-70.

PETRESCU R. V.; PETRESCU F. I.; POPESCU N. (2007), Determining Gear Efficiency, In Gear Solutions magazine, USA, p. 19-28, March. Available on: http://www.gearsolutions.com/media//uploads/assets//PDF/Magazines/GearSolutions 0307.pdf

PETRESCU, F. I. (2012), Teoria mecanismelor - Curs si aplicatii (editia a doua), Create Space publisher, USA, September, ISBN 978-1-4792-9362-9, 284 pages, Romanian version. Available on: http://www.amazon.com/Teoria-Mecanismelor-Cursaplicatii-Romanian/dp/1479293628

REY G. G. (2013), Influencia de la lubricacion en la eficiencia de engranajes de tornillo sinfin, Ingineria Mecanica journal, ISSN 1815-5944, v. 16, n. 1, p. 13-21. Available on: http://www.redalyc.org/articulo.oa?id=225125743002

STOICA, I. A. (1977), Interferenţa roţilor dinţate. Editura DACIA, Cluj-Napoca. 